\title{
Carob pulp preparation rich in insoluble dietary fibre and polyphenols increases plasma glucose and serum insulin responses in combination with a glucose load in humans
}

\author{
Sindy Gruendel ${ }^{1} \dagger$, Baerbel Otto $^{2} \dagger$, Ada L. Garcia ${ }^{1}$, Karen Wagner ${ }^{1}$, Corinna Mueller ${ }^{1}$, \\ Martin O. Weickert ${ }^{3,4}$, Walter Heldwein ${ }^{2}$ and Corinna Koebnick ${ }^{1,5 *}$ \\ ${ }^{1}$ Dietary Fibre and the Metabolic Syndrome Research Group, German Institute of Human Nutrition Potsdam-Rehbruecke, \\ Nuthetal, Germany \\ ${ }^{2}$ Medical Department-Innenstadt, University Hospital, Munich, Germany \\ ${ }^{3}$ Department of Clinical Nutrition, German Institute of Human Nutrition Potsdam-Rehbruecke, Nuthetal, Germany \\ ${ }^{4}$ Department of Endocrinology, Diabetes and Nutrition, Charité-University-Medicine, Campus Benjamin Franklin, \\ Berlin, Germany \\ ${ }^{5}$ Department of Preventive Medicine, University of Southern California, Los Angeles, USA
}

(Received 22 August 2006 - Revised 19 December 2006 - Accepted 18 January 2007)

\begin{abstract}
Dietary fibre consumption is associated with improved glucose homeostasis. In contrast, dietary polyphenols have been suggested to exert both beneficial and detrimental effects on glucose and insulin metabolism. Recently, we reported that a polyphenol-rich insoluble dietary fibre preparation from carob pulp (carob fibre) resulted in lower postprandial acylated ghrelin levels after a liquid meal challenge test compared with a control meal without supplementation. The effects may, however, differ when a different food matrix is used. Thus, we investigated the effects of carob fibre on glucose, insulin and ghrelin responses in healthy humans in combination with a glucose load. In a randomized single-blind cross-over study involving twenty healthy subjects (aged 22-62 years), plasma glucose, total and acylated ghrelin, and serum insulin were repeatedly assessed before and after the ingestion of $200 \mathrm{ml}$ water with $50 \mathrm{~g}$ glucose and $0,5,10 \mathrm{or} 20 \mathrm{~g}$ carob fibre over a period of $180 \mathrm{~min}$. The intake of 5 and $10 \mathrm{~g}$ carob fibre increased the plasma glucose by $47 \%$ and $64 \%(P<0 \cdot 001)$, and serum insulin by 19.9 and $24.8 \%(P<0 \cdot 001)$, compared with the control. Plasma acylated ghrelin concentrations did not change significantly after the consumption of carob-enriched glucose solution. Total ghrelin decreased only after $10 \mathrm{~g}$ carob fibre $(P<0.001)$ compared with control. In conclusion, we showed that polyphenol-rich carob fibre, administered within a water-glucose solution, increases postprandial glucose and insulin responses, suggesting a deterioration in glycaemic control.
\end{abstract}

Carob: Insoluble dietary fibre: Polyphenols: Glucose: Insulin: Ghrelin

Dietary fibre, a complex group of substances with various physicochemical properties, has been shown to improve glucose homeostasis (Kimmel et al. 2000; Chau et al. 2004) and has consistently been linked to a reduced risk of type 2 diabetes and cardiovascular disease in population studies. In short-term intervention studies, viscous soluble dietary fibre has been shown to improve glucose handling after a meal via decreased absorption rates of carbohydrates (Tabatabai $\& \mathrm{Li}, 2000$ ). Soluble fibre has also been shown to reduce postprandial insulin responses (Del Toma et al. 1988). On the other hand, the consumption of highly purified insoluble dietary fibre has been shown to accelerate the acute insulin response directly after consumption, and to further enhance postprandial carbohydrate handling the following day upon the ingestion of a control meal, which has been explained by improved whole-body insulin sensitivity (Weickert et al. 2006a).
In whole foods such as cereals, other bioactive substances beyond dietary fibre might contribute to metabolic control. We recently showed that the consumption of a standardized liquid meal enriched with a polyphenol-rich insoluble dietary fibre preparation from carob pod (carob fibre) decreased serum triacylglycerols and NEFA, as well as the secretion of the gastrointestinal hormone ghrelin (Gruendel et al. 2006). Ghrelin is a gut hormone with multiple functions, produced primarily in the stomach to regulate energy balance. Acylated ghrelin is one of two major forms of ghrelin; it is known to exhibit most of the biological activities of ghrelin, including orexigenic effects via hypothalamic pathways (Korbonits et al. 2004). Ghrelin secretion has been controversially associated with insulin and glucose responses. Postprandial ghrelin secretion is affected by the caloric, macronutrient and fibre content of ingested meals (Erdmann et al. 2004; Weickert et al. 2006b), but preprandial secretion is not modified.

Corresponding author: Dr Corinna Koebnick, fax +331323422 4103, email koebnick@usc.edu

$\dagger$ Both authors contributed equally. 
Our recent findings indicated that carob fibre rich in polyphenols decreases ghrelin secretion after a liquid meal challenge test containing $34 \%$ energy as fat. The effects of carob fibre within a differing food matrix are, however, unknown. Additionally, polyphenols have been shown to have effects on glucose handling (Thompson et al. 1984; Kamei et al. 2002).

Therefore, the aim of the present study was to investigate the dose-dependent effects of carob fibre on postprandial glucose handling and ghrelin response when administered in combination with a glucose load.

\section{Subjects and methods}

\section{Subjects}

Twenty healthy adults (twelve women and eight men) participated in the study. Inclusion criterion was a BMI (= body weight $(\mathrm{kg}) /$ height $\left.(\mathrm{m})^{2}\right)$ within the normal range $(18.5-$ $25 \mathrm{~kg} / \mathrm{m}^{2}$ ). Exclusion criteria were a history of chronic diseases, dyslipidaemia (fasting triglycerides $>2.3 \mathrm{mmol} / \mathrm{l}$, total cholesterol $>5.2 \mathrm{mmol} / \mathrm{l}, \quad$ LDL-cholesterol $>4.0 \mathrm{mmol} / \mathrm{l}$, HDL-cholesterol $<0.9 \mathrm{mmol} / \mathrm{l})$, impaired glucose tolerance (fasting glucose $>6.1 \mathrm{mmol} / \mathrm{l}$ ), intentional weight loss within 3 months prior to the study, extreme sports and any medication influencing glucose or lipid metabolism. The ethics committee of the University of Potsdam approved the study protocol. All participants gave written consent before starting the study.

\section{Research design}

The study was conducted as a randomized cross-over trial consisting of four sessions lasting $180 \mathrm{~min}$ each, separated by intervals of at least 1 week. Subjects entered our facilities between 07.30 and 08.00 hours after an overnight fast. Subjects consumed $200 \mathrm{ml}$ water with $50 \mathrm{~g}$ glucose (Dextropur; Maizena Diät GmbH, Heilbronn, Germany) and 0, 5, 10 or $20 \mathrm{~g}$ carob fibre provided in randomized order. The energy content of the glucose preparation was $857 \mathrm{~kJ}$. The carob fibre contained $5.8 \mathrm{~g}$ simple carbohydrates (glucose, fructose and sucrose), $5 \cdot 2 \mathrm{~g}$ protein and $0 \cdot 2 \mathrm{~g}$ fat per $100 \mathrm{~g}$. The total dietary fibre content was $74.6 \mathrm{~g}$ per $100 \mathrm{~g}$ carob pulp preparation, corresponding to $68.4 \mathrm{~g}$ insoluble and $6.2 \mathrm{~g}$ soluble fibre. The total polyphenol content of the preparation was $2.8 \mathrm{~g}$ per $100 \mathrm{~g}$. The major polyphenol compounds of carob fibre are gallic acid, gallotannins and flavonol glycosides. The energy contribution of the carob fibre enrichment was negligible with respect to the total energy intake of the glucose load $(1.2 \%$ for $5 \mathrm{~g}, 2.3 \%$ for $10 \mathrm{~g}$, and $4.7 \%$ for $20 \mathrm{~g}$ carob fibre). Participants were blinded to the fibre content of the test meals, but due to taste and colour it was possible to distinguish the meals. Participants were not allowed to consume food or beverages during the examinations.

\section{Blood sampling and biochemical analysis}

An indwelling catheter was inserted into an antecubital vein of the subjects, and blood samples were collected before and 15 , $30,45,60,75,90,120$ and 180 min after administration of the test meal.
For plasma separation, blood was collected in EDTA-containing tubes and immediately centrifuged. To obtain serum, blood was collected in tubes containing a serum clot activator and allowed to clot. Plasma and serum samples were centrifuged at $1500 \mathrm{~g}$ for $10 \mathrm{~min}$ at $4^{\circ} \mathrm{C}$; plasma and serum supernatants were obtained and stored at $-40^{\circ} \mathrm{C}$. For measurement of ghrelin, plasma was collected in tubes containing EDTA and $500 \mathrm{U}$ aprotinin (Bayer, Leverkusen, Germany) per millilitre whole blood. For stabilization plasma supernatant was acidified with $1 \mathrm{M}-\mathrm{HCl}$ (Merck, Darmstadt, Germany) and stored at $-40^{\circ} \mathrm{C}$.

Plasma glucose was measured by the hexokinase method using commercially available colorimetric reagents (ABX Diagnostics Glucose; ABX Diagnostics, Montpellier, France) with an intra-assay CV of $1.2 \%$. Serum insulin was determined by Mercodia Insulin ELISA assay (Mercodia, Uppsala, Sweden) with an intra-assay CV of $2.6 \%$. Homoeostasis model assessment for insulin resistance (HOMA-IR) was calculated as [fasting insulin $(\mathrm{mU} / \mathrm{l}) \times$ fasting glucose $(\mathrm{mmol} / \mathrm{l}) /$ 22.5].

Plasma total ghrelin was measured by a commercial RIA, which utilizes ${ }^{125}$ I-labelled ghrelin and a ghrelin antiserum to determine the concentration of total ghrelin in the plasma by a double-antibody/polyethyleneglycol technique (Linco Research, Missouri, MO, USA). The RIA was specific for the C-terminal portion of ghrelin (amino acids 14-28). The sensitivity of the method was $93 \mathrm{pg} / \mathrm{ml}$. There were no cross-reactions with ghrelin 1-10, motilin-related peptide, glucagon, leptin or insulin. The intra- and inter-assay CV were $10.0 \%$ and $14.7 \%$, respectively, and the percentage recovery was calculated as $96 \%$. Plasma acylated ghrelin determination was performed using RIA that recognized the $\mathrm{N}$-terminal portion of the ghrelin molecule (amino acids $1-10)$. It utilizes ${ }^{125}$ I-labeled ghrelin as a tracer and an antibody (raised in guinea pigs) that is specific for the octanoyl group on serine 3 (Linco Research). The sensitivity of the method was $7.8 \mathrm{pg} / \mathrm{ml}$. There were no cross-reactions with ghrelin 14-28, motilin-related peptide, glucagon, leptin or insulin. The intra- and inter-assay CV were $6.7 \%$ and $9.6 \%$, respectively. Percentage recovery was calculated as $114 \%$.

Serum total cholesterol was analysed using the cholesterol oxidase phenol 4-aminoantipyrine peroxidase (CHOD/PAP) method (ABX Diagnostics Cholesterol; ABX Diagnostics) with an intra-assay CV of $0.82 \%$. Serum HDL-cholesterol was analysed using a colorimetric test (ABX Diagnostics HDL Cholesterol Direct; ABX Diagnostics) with an intraassay CV of $1.29 \%$. LDL-cholesterol was calculated according to Friedewald (Friedewald et al. 1972).

\section{Statistical analyses}

All statistical procedures were made using SPSS for Windows 11.5 (SPSS Inc., Chicago, IL, USA). Baseline characteristics are shown as mean and standard deviations, whereas figures show means and their standard errors. Time series data for all parameters and for each subject were normalized to baseline values, which was defined as time $=0 \mathrm{~min}$. Differences in response to the glucose load were tested by a mixed linear model with time, carob concentration and carob concentration $\times$ time as fixed factors and subject as a random factor. Post hoc comparisons of carob fibre concentrations with the 
control treatment were adjusted according to the Bonferroni method. Percentage changes in the postprandial response of the outcome variables were calculated based on the response to the control treatment as a reference. The mean percentage increase or decrease was calculated for the time point with the highest (for glucose, insulin and triacylglycerols) or lowest (for ghrelin and NEFA) time points of the curves. A probability $P<0.05$ was considered as significant.

\section{Results}

Twenty healthy adults aged 29.4 (SD 2.6) years (range 22-62 years) and with a BMI of 23.0 (SD $0.5 \mathrm{~kg} / \mathrm{m}^{2}$ (range 20.4$27.5 \mathrm{~kg} / \mathrm{m}^{2}$ ) participated in the study. Fasting plasma glucose was $5 \cdot 1(\mathrm{SD} 0 \cdot 7) \mathrm{mmol} / \mathrm{l}$, and fasting serum insulin was $43 \cdot 7$ (SD 25.8) pmol/l.

After the glucose load, the plasma glucose and insulin concentrations increased $(P<0 \cdot 001)$. The consumption of carob fibre significantly affected the glucose and insulin responses $(P<0.001)$. After the consumption of test meals with 5 and $10 \mathrm{~g}$ carob fibre, plasma glucose increased significantly, up to $147 \%$ and $164 \%$, respectively $(P<0.001)$, compared with the glucose control drink. The consumption of $20 \mathrm{~g}$ carob fibre did not result in a significant increase in plasma glucose compared with control $(P=0.976)$ (Fig. 1(A)).

After glucose load, acylated $(P<0.001)$ but not total plasma ghrelin decreased rapidly. No effects of carob fibre consumption on acylated plasma ghrelin were, however, observed (Fig. 1(D)). Total plasma ghrelin was slightly but significantly decreased compared with controls $(P=0.001)$ after the consumption of $10 \mathrm{~g}$ carob fibre. No effects were observed after 5 and $20 \mathrm{~g}$ carob fibre (Fig. 1(C)).

\section{Discussion}

The major finding of the present study was a significantly increased glucose and insulin response after consumption of a phenol-rich insoluble dietary fibre preparation made from carob, after a glucose load enriched with up to $10 \mathrm{~g}$ carob fibre, compared with a control. The increase in glucose response failed to reach statistical significance after the consumption of $20 \mathrm{~g}$ carob fibre, which might be explained by the higher variance in glucose response in the present experiment. In contrast to prior observations, carob fibre consumption did not alter the response of acylated ghrelin after a glucose load, with only minor changes in total ghrelin after $10 \mathrm{~g}$ carob fibre compared with control. Using a different food matrix (i.e. a liquid meal challenge test with $34 \%$ of energy as fat), we recently reported that the consumption of insoluble dietary fibre from carob pulp significantly decreased postprandial acylated ghrelin and markedly improved the postprandial lipid response and fat oxidation (Gruendel et al. 2006).

Dietary fibre affects postprandial glucose and lipid responses by several mechanisms. Dietary fibre increases gastric distension, which has been suggested to trigger afferent vagal signals of fullness in some (Krotkiewski, 1985; Saltzman \& Roberts, 1997; Howarth et al. 2001) but not all studies (Howarth et al. 2003). Some forms of soluble dietary fibre increase the viscosity of the intestinal tract contents, delay gastric emptying (Wolever \& Jenkins, 1993; Bonfield,
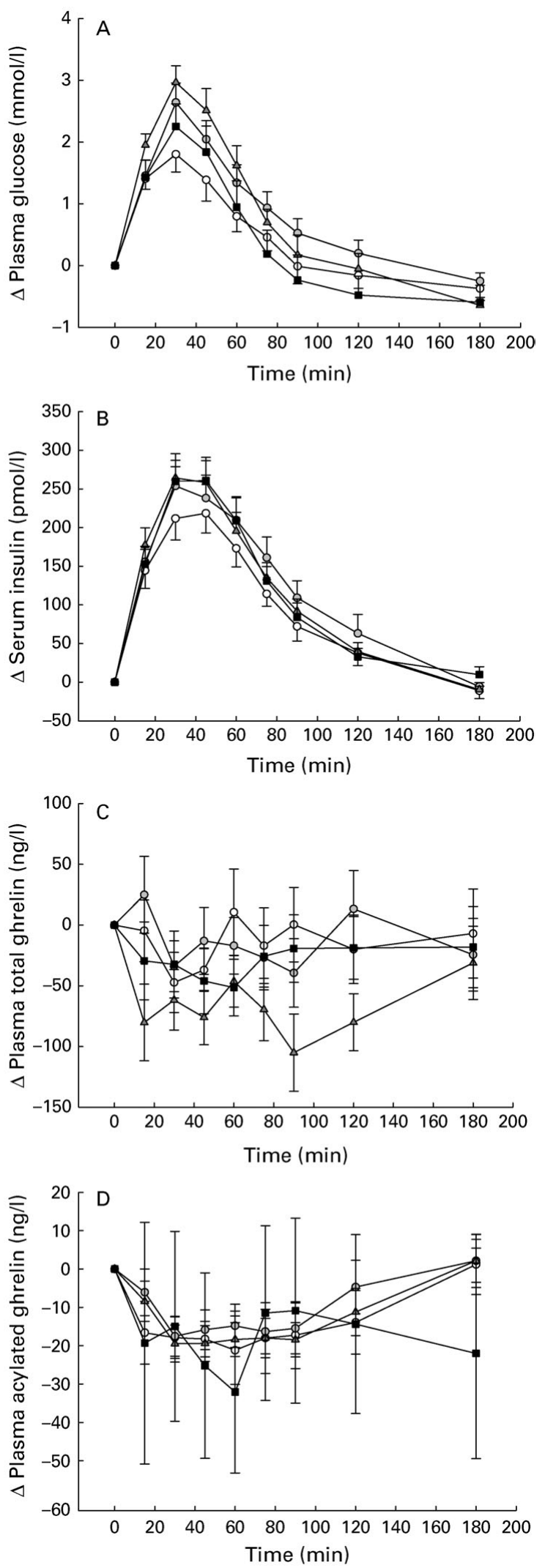

Fig. 1. Changes in plasma glucose (A), serum insulin (B), plasma total ghrelin (C), and plasma acylated ghrelin (D) as means and their standard errors relative to baseline after a test meal with or without carob fibre $(200 \mathrm{ml}$ water $+50 \mathrm{~g}$ glucose $+0\left(-\mathrm{O}_{-}\right), 5\left(-\mathrm{O}_{-}\right), 10\left(-\Delta_{-}\right)$or $20(-\mathbf{-}) \mathrm{g}$ carob) in healthy subjects $(n 20)$. 
1995), and decrease the absorption of macronutrients by inhibiting resorption (Slavin, 2005). In the present study, however, most of the effects described above are unlikely to be involved. First, effects of mastication were eliminated as a liquid solution supplemented with carob fibre powder was administered. Second, carob fibre mainly consisted of insoluble dietary fibre, which is unlikely to increase viscosity. Therefore, the effects of carob fibre could have been mediated through mechanisms related to the polyphenol content.

However, studies on the glucose- and insulin-regulating activities of polyphenols are controversial. It has been suggested that polyphenols exert both beneficial and detrimental effects on glucose metabolism. Polyphenols have been shown to lower blood glucose responses (Thompson et al. 1984) and to inhibit carbohydrate digestion by modulating the activity of $\alpha$-amylase (Kandra et al. 2004; McDougall et al. 2005). Cell culture studies using 3T3-L1 adipocytes and MCF-7 breast cancer cells have shown that polyphenols inhibit the activity of phosphoinositide 3-kinase, a key regulator of insulin-induced GLUT4 translocation (Harmon \& Patel, 2003, 2004). In isolated rat adipocytes, some polyphenols inhibited glucose uptake (Strobel et al. 2005). It has also been suggested that GLUT4 interacts directly with flavonoids and that GLUT transporters are involved in flavonoid incorporation into cells (Strobel et al. 2005). In contrast, other polyphenols may also stimulate glucose uptake in 3T3-L1 adipocytes via an insulin-independent tyrosine kinase pathway (Kamei et al. 2002). In addition, it has been recently described that increased insulin sensitivity resulting from resistant starch consumption was not mediated by GLUT4. Therefore we cannot exclude the idea that the effects observed in the present study were mediated by the polyphenol contents rather than by dietary fibre per se.

Taken together, studies investigating the effects of polyphenols may partly explain our observations. The consumption of carob fibre appears to increase postprandial glucose and insulin responses in combination with a monosaccharide (glucose) load, an effect that is not observed when carob fibre is ingested within a liquid meal containing disaccharides, protein and fat (Gruendel et al. 2006). The mechanisms of action in the present study remain, however, speculative.

The lack of marked effects on total and acylated ghrelin responses observed in the present study is in contrast with a previous one (Gruendel et al. 2006) and may be explained by the background food matrix. It has been shown that postprandial ghrelin responses are influenced by the macronutrient content of food. Dietary carbohydrate ingestion produced more pronounced decreases in total ghrelin concentration when compared with protein- and fat-rich meals (Erdmann et al. 2003; Monteleone et al. 2003). Recently, it has been shown that ghrelin secretion is suppressed by the presence of fat in the small intestine. Moreover, the fat-induced suppression of ghrelin is dependent on fat digestion (FeinleBisset et al. 2005).

The decrease in acylated ghrelin caused by carob fibre consumption as observed after the liquid meal challenge test was not related to glucose and insulin responses and therefore, might be related to alterations in lipid metabolism (Gruendel et al. 2006). In the present study, carob fibre consumption after a glucose load did not result in relevant changes of plasma ghrelin concentration, a finding that we are unable to explain. In contrast, other studies have demonstrated that, after a glucose load, ghrelin levels decreased by $28 \%$. However, the short-term parenteral administration of glucose and insulin in physiological doses did not suppress ghrelin levels, suggesting that changes in plasma insulin and glucose were not responsible for changes in ghrelin levels after food intake.

The results of the present study must be interpreted with some caution because the water-glucose solution used here is not comparable to the food matrix of a normal mixed diet. Nevertheless, the data suggest that an excessive consumption of carob fibre may exert adverse effects on glucose handling. This might be relevant for the potential enrichment of sugar-sweetened beverages by carob fibre. Further studies within differing background meals are needed to confirm these results. Investigating the long-term effects of carob fibre on glucose handling and insulin sensitivity might be of further interest.

In conclusion, the ingestion of carob fibre rich in polyphenols increased postprandial glucose and insulin concentrations, suggesting an exacerbated glycaemic control in combination with a water-glucose solution, but did not markedly affect postprandial ghrelin concentrations. The mechanisms of this phenomenon remain to be investigated in future studies.

\section{Acknowledgements}

We thank Andreas Wagner and Minerva Petrovitsch for the excellent technical assistance.

\section{References}

Bonfield C (1995) Dietary fiber and weight management. In Dietary Fiber in Health and Disease, pp. 459-465 [D Kritchevsky and C Bonfield, editors]. St. Paul, MN: Eagan Press.

Chau CF, Chen CH \& Lee MH (2004) Characterization and physicochemical properties of some potential fibres derived from Averrhoa carambola. Nahrung 48, 43-46.

Del Toma E, Lintas C, Clementi A \& Marcelli M (1988) Soluble and insoluble dietary fibre in diabetic diets. Eur J Clin Nutr 42, 313-319.

Erdmann J, Lippl F \& Schusdziarra V (2003) Differential effect of protein and fat on plasma ghrelin levels in man. Regul Pept 116, $101-107$.

Erdmann J, Topsch R, Lippl F, Gussmann P \& Schusdziarra V (2004) Postprandial response of plasma ghrelin levels to various test meals in relation to food intake, plasma insulin, and glucose. J Clin Endocrinol Metab 89, 3048-3054.

Feinle-Bisset C, Patterson M, Ghatei MA, Bloom SR \& Horowitz M (2005) Fat digestion is required for the suppression of ghrelin and stimulation of peptide $\mathrm{YY}$ and pancreatic polypeptide secretion by intraduodenal lipid. Am J Physiol Endocrinol Metab 289, E948-953.

Friedewald WT, Levy RI \& Fredrickson DS (1972) Estimation of the concentration of low-density lipoprotein cholesterol in plasma, without use of the preparative ultracentrifuge. Clin Chem 18, 499-502.

Gruendel S, Garcia AL, Otto B, Mueller C, Steiniger J, Weickert MO, Speth M, Katz N \& Koebnick C (2006) Carob pulp preparation rich in insoluble dietary fiber and polyphenols enhances lipid oxidation and lowers postprandial acylated ghrelin in humans. J Nutr 136, $1533-1538$. 
Harmon AW \& Patel YM (2003) Naringenin inhibits phosphoinositide 3-kinase activity and glucose uptake in 3T3-L1 adipocytes. Biochem Biophys Res Commun 305, 229-234.

Harmon AW \& Patel YM (2004) Naringenin inhibits glucose uptake in MCF-7 breast cancer cells: a mechanism for impaired cellular proliferation. Breast Cancer Res Treat 85, 103-110.

Howarth NC, Saltzman E, McCrory MA, Greenberg AS, Dwyer J, Ausman L, Kramer DG \& Roberts SB (2003) Fermentable and nonfermentable fiber supplements did not alter hunger, satiety or body weight in a pilot study of men and women consuming selfselected diets. J Nutr 133, 3141-3144.

Howarth NC, Saltzman E \& Roberts SB (2001) Dietary fiber and weight regulation. Nutr Rev 59, 129-139.

Kamei R, Kitagawa Y, Kadokura M, Hattori F, Hazeki O, Ebina Y, Nishihara T \& Oikawa S (2002) Shikonin stimulates glucose uptake in 3T3-L1 adipocytes via an insulin-independent tyrosine kinase pathway. Biochem Biophys Res Commun 292, 642-651.

Kandra L, Gyemant G, Zajacz A \& Batta G (2004) Inhibitory effects of tannin on human salivary alpha-amylase. Biochem Biophys Res Commun 319, 1265-1271.

Kimmel SE, Michel KE, Hess RS \& Ward CR (2000) Effects of insoluble and soluble dietary fiber on glycemic control in dogs with naturally occurring insulin-dependent diabetes mellitus. $J$ Am Vet Med Assoc 216, 1076-1081.

Korbonits M, Goldstone AP, Gueorguiev M \& Grossman AB (2004) Ghrelin - a hormone with multiple functions. Front Neuroendocrinol 25, 27-68.

Krotkiewski M (1985) Use of fibres in different weight reduction programs. In Current Topics on Nutrition and Disease: Dietary Fiber and Obesity, pp. 85-109 [P Bjorntorp, GV Vahouny and D Kritchevsky, editors]. New York: Alan R. Liss.

McDougall GJ, Shapiro F, Dobson P, Smith P, Blake A \& Stewart D (2005) Different polyphenolic components of soft fruits inhibit alpha-amylase and alpha-glucosidase. J Agric Food Chem 53, $2760-2766$.

Monteleone P, Bencivenga R, Longobardi N, Serritella C \& Maj M (2003) Differential responses of circulating ghrelin to high-fat or high-carbohydrate meal in healthy women. J Clin Endocrinol Metab 88, 5510-5514.

Saltzman E \& Roberts SB (1997) Soluble fiber and energy regulation. Current knowledge and future directions. Adv Exp Med Biol 427, 89-97.

Slavin JL (2005) Dietary fiber and body weight. Nutrition 21, 411-418.

Strobel P, Allard C, Perez-Acle T, Calderon R, Aldunate R \& Leighton F (2005) Myricetin, quercetin and catechin-gallate inhibit glucose uptake in isolated rat adipocytes. Biochem $J \mathbf{3 8 6}$, 471-478.

Tabatabai A \& Li S (2000) Dietary fiber and type 2 diabetes. Clin Excell Nurse Pract 4, 272-276.

Thompson LU, Yoon JH, Jenkins DJ, Wolever TM \& Jenkins AL (1984) Relationship between polyphenol intake and blood glucose response of normal and diabetic individuals. Am J Clin Nutr 39, 745-751.

Weickert MO, Mohlig M, Schofl C, Arafat AM, Otto B, Viehoff H, Koebnick C, Kohl A, Spranger J \& Pfeiffer AF (2006a) Cereal fiber improves whole-body insulin sensitivity in overweight and obese women. Diabetes Care 29, 775-780.

Weickert MO, Spranger J, Holst JJ, Otto B, Koebnick C, Mohlig $\mathrm{M}$ \& Pfeiffer AF (2006b) Wheat-fibre induced changes of postprandial peptide YY (PYY) and ghrelin responses are not associated with acute alterations of satiety. $B r J$ Nutr 96, 795-798.

Wolever T \& Jenkins DJA (1993) Effects of dietary fiber and foods on carbohydrate metabolism. In CRC Handbook of Dietary Fiber in Human Nutrition, pp. 111-152 [G Spiller, editor]. Boca Raton, FL: CRC Press. 